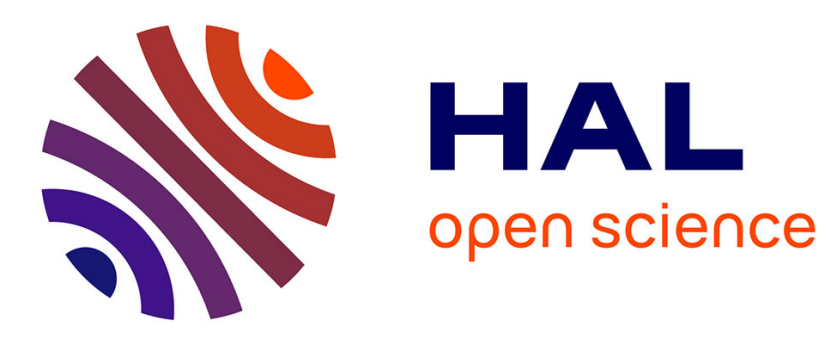

\title{
Ultimate Bounds and Robust Invariant Sets for Linear Systems with State-Dependent Disturbances
}

\author{
Sorin Olaru, Vasso Reppa
}

\section{To cite this version:}

Sorin Olaru, Vasso Reppa. Ultimate Bounds and Robust Invariant Sets for Linear Systems with State-Dependent Disturbances. Sorin Olaru, Alexandra Grancharova, Fernando Lobo Pereira. Developments in Model-Based Optimization and Control, 464, Springer, pp.339-359, 2015, Lecture Notes in Control and Information Sciences, 9783319266855. 10.1007/978-3-319-26687-9_16 . hal-01260190

\section{HAL Id: hal-01260190 \\ https://hal-centralesupelec.archives-ouvertes.fr/hal-01260190}

Submitted on 2 Mar 2018

HAL is a multi-disciplinary open access archive for the deposit and dissemination of scientific research documents, whether they are published or not. The documents may come from teaching and research institutions in France or abroad, or from public or private research centers.
L'archive ouverte pluridisciplinaire HAL, est destinée au dépôt et à la diffusion de documents scientifiques de niveau recherche, publiés ou non, émanant des établissements d'enseignement et de recherche français ou étrangers, des laboratoires publics ou privés. 


\title{
Ultimate bounds and robust invariant sets for linear systems with state-dependent disturbances
}

Sorin Olaru and Vasso Reppa

\begin{abstract}
The objective of this chapter is to present a methodology for computing robust positively invariant sets for linear, discrete time invariant systems that are affected by additive disturbances, with the particularity that these disturbances are subject to state-dependent bounds. The proposed methodology requires less restrictive assumptions compared to similar established techniques, while it provides the framework for determining the state-dependent (parameterized) ultimate bounds for several classes of disturbances. The added value of the proposed approach is shown by formulating an optimization-based detection of the mode of functioning in a switching system.
\end{abstract}

\section{Introduction}

The interest in analysis and control design for linear dynamics with set constrained disturbances is a mature subject in control theory [5, 12]. The analysis of dynamical systems affected by state and control dependent disturbances is also a wellestablished subject which can be traced back to the early 70's [20,29] mainly in the stochastic control systems framework and latter in the works on the mismatched uncertainties [4, 19]. We recall for example some historical observations made in [20] stating that "the general conclusion is that control-dependent noise calls for conser-

Sorin Olaru and Vasso Reppa

Laboratory of Signals and Systems (UMR CNRS 8506), CentraleSupélec-CNRS-Univ.

Paris-Sud, Gif-Sur-Yvette, 91192, France e-mail: sorin.olaruecentralesupelec.fr; vasiliki.reppa@centralesupelec.fr 
vative control (small gains) while state-dependent noise calls for vigorous control (large gains)".

Results dealing with both set-theoretic notions and state-dependent disturbances can be mentioned in various studies dedicated to viability theory [1]; propagation of parametric uncertainties [3]; control of system with uncertainties in the parameters as well as in the input itself [18]; ultimate boudedness control via set-induced Lyapunov functions [7]; reachability analysis [23], maximal invariant sets [10, 25]. In the present paper, we are interested in the characterization of the ultimateboundedness of linear dynamical systems affected by additive disturbances with the particularity that these disturbances are subject to state-dependent bounds.

From the theoretical point of view, there are different connections with the mature literature on minimal invariant sets of dynamic systems with bounded disturbances $[13,15,17,22]$ and in a broader sense to the set-theoretic methods in control $[6,8,9]$. From a practical standpoint, the characterization of positive invariant sets can be used for diagnosis. Recently, the set-theoretic methods have been used in model-based fault diagnosis (FD) and the design of fault tolerant control (FTC) laws [28]. The positive invariance enables the analysis and offers guarantees for FD/FTC performance, that is robustness, fault detectability and isolability, and fault tolerance $[11,21,24,26,27]$ under strict set-inclusion or set-separation conditions.

The goal and the main contribution of this chapter is to establish a methodology for computing robust positively invariant (RPI) sets for a class of discrete linear systems, affected by disturbances bounded by a state dependent function. In order to highlight the contribution, in Section 2 we present some established methodologies for computing state-independent RPI sets and extensions for state-dependent RPI sets and ultimate bounds, which however require more restrictive assumptions compared to those used in the present work. Then, in Section 3 we describe a new approach for the computation of the state dependent (parameterized) RPI sets, providing some examples for illustrating both the applicability and limitations of the proposed methodology. In Section 4, we initially discuss the design of the parameterized RPI sets for some special classes of systems and the state-dependent function that bounds the additive system disturbances. Then, we present how the computation of parameterized sets can be formulated as an optimization problem that can be applied for detecting the system mode switching (e.g. due to a fault). Section 5 offers some concluding remarks of this work and some future directions.

Notation: $\mathbb{Z}$ is the set of all integers. $\mathbb{Z}_{+}$is the set of all nonnegative integers and $\mathbb{Z}_{\left[k_{0}, k_{1}\right]}$ the set of nonnegative integers in the interval $\left[k_{0}, k_{1}\right] . \mathbb{R}^{n}$ is the $n$ dimensional Euclidean space with $\|$.$\| denoting the prescribed norm (Euclidean$ norm for simplicity). The closed convex hull of a set $S$ will be denoted $C o n v\{S\}$. 
The Minkowski sum of two sets $S_{1}, S_{2} \subset \mathbb{R}^{n}$ will be denoted by $S_{1} \oplus S_{2}$. The image of $S \subset \mathbb{R}^{n}$ via $g: \mathbb{R}^{n} \rightarrow \mathbb{R}^{m}$ is described by the set $g(S)=\{g(x): x \in S\}$.

Given a function $f: \mathbb{R}^{n} \rightarrow \mathbb{R}$, its level set (contour) for $c \in \mathbb{R}$ is defined as

$$
\mathcal{L}_{f}(c)=\left\{x \in \mathbb{R}^{n}: f(x)=c\right\},
$$

while its sublevel set for given $c \in \mathbb{R}$ is described as

$$
\mathcal{L}_{f}^{-}(c)=\left\{x \in \mathbb{R}^{n}: f(x) \leq c\right\} .
$$

Definition 1. A set $S \subset \mathbb{R}^{n}$ is star-shaped in $\bar{x} \in \mathbb{R}^{n}$ if for any point $x \in S$ and $0 \leq \alpha \leq 1$ it holds that $\alpha x+(1-\alpha) \bar{x} \in S$.

Definition 2. A function $f: \mathbb{R}^{n} \rightarrow \mathbb{R}_{+}$is called increasing from $\bar{x} \in \mathbb{R}^{n}$ if any sublevel set $\mathcal{L}_{f}^{-}(c), c \in \mathbb{R}_{+}$is a star-shaped set in $\bar{x}$.

\section{Background}

\subsection{Problem formulation}

Consider a discrete time linear uncertain system:

$$
x_{k+1}=A x_{k}+B w_{k}
$$

where $x_{k} \in \mathbb{R}^{n}$ is the state vector at the time $k \in \mathbb{Z}_{+}$and $A \in \mathbb{R}^{n \times n}, B \in \mathbb{R}^{n}$. It is considered that dynamics (3) correspond to a closed loop system, for which the exponential stability is guaranteed in the disturbance-free case according to the following assumption:

Assumption 1 The matrix $A$ is Schur (all the eigenvalues are inside the unit circle).

In the present chapter we concentrate on the case of a matrix $B$ represented by a column vector, which is related to a signal $w_{k} \in \mathbb{R}$ representing the additive disturbance. The main characteristic of the additive disturbance will be its boundedness by a state dependent function $f: \mathbb{R}^{n} \rightarrow \mathbb{R}_{+}$such that:

$$
\left\|w_{k}\right\| \leq f\left(x_{k}\right)
$$


Definition 3. Let us consider the solution $x_{k}: \mathbb{Z}_{+} \rightarrow \mathbb{R}^{n}$ of (3) denoted as $x_{k}=$ $x\left(x_{0}, \mathbf{w}^{0: k-1}\right)$ for a given initial condition $x_{0}$ and disturbance sequence $\mathbf{w}^{0: k-1}=$ $\left(w_{0}, \ldots, w_{k-1}\right) \in \mathbb{R}^{k}$ satisfying (4). We say that:

- $x_{k}$ is bounded if there exists a positive constant $d\left(x_{0}\right)<\infty$ such that the inequality $\left\|x\left(x_{0}, \mathbf{w}^{0: k-1}\right)\right\| \leq d\left(x_{0}\right)$ holds for all $k \in \mathbb{Z}_{+}$. If the initial condition $x_{0}$ can be chosen arbitrarily large then the solutions of (3) are globally bounded. ${ }^{1}$

- $x_{k}$ is ultimately bounded if there exists a bounded set $S \subset \mathbb{R}^{n}$, possibly dependent on $x_{0}$, and a nonnegative integer $T\left(x_{0}, S\right)<\infty$, such that $x_{k} \in S$ for all $k \geq T\left(x_{0}, S\right)$.

- The bounded set $S$ represents an ultimate bound for the trajectories initiated in $x_{0}$ if the sequence of sets $X_{0}=\left\{x_{0}\right\}, X_{k+1}=A X_{k} \oplus B f\left(X_{k}\right)$ satisfies $^{2}$

$$
\limsup _{k \rightarrow \infty} X_{k} \subset S \text {. }
$$

The set $S$ is a global ultimate bound if it is an ultimate bound for any $x_{0} \in \mathbb{R}^{n}$.

The problem to be considered in this paper can be outlined as follows:

Objective 1: Find sufficient conditions for ultimate boundedness of the state trajectories satisfying (3) taking into account (4).

Objective 2: Describe the parameterization of ultimate bounds with respect to the initial conditions, when these ultimate bounds are not global. In this respect, we seek to express ultimate bounds in terms of compact and convex sets.

From the analysis point of view, the robust positive invariance of a set with respect to the system dynamics is another important notion in the present work.

Definition 4. (RPI set) A set $\Omega \subset \mathbb{R}^{n}$ is a robust positively invariant (RPI) set with respect to (3) if $A x+B w \in \Omega$ for all $x \in \Omega$ and for all $w$ satisfying $\|w\| \leq f(x)$.

Even if an ultimate bound set is not à priori RPI, we will be interested in finding sets which are both RPI and ultimate bounds for the state trajectories.

\footnotetext{
${ }^{1}$ Often the notion of global boundedness is complemented by the attribute uniform to emphasize the possible dependence of the bound on the initial condition $x_{0}$ but not on the initial moment in time. This addition is superfluous for time-invariant dynamics and will be abandoned here.

${ }^{2}$ The set $S$ is a proper superset of $\lim _{\sup _{k \rightarrow \infty}} X_{k}$. The meaning of the outer limit (limsup) is particular in this context as it is understood in a set-theoretic framework [2] as the set of cluster points of sequences $x\left(x_{0}, \mathbf{w}^{0: k-1}\right) \in X_{k}$.
} 


\subsection{A detour on the state-independent disturbance bounds case}

Strong results are available with respect to the RPI sets description in the case of state-independent bounds on disturbance. Let us consider the class of dynamics:

$$
x_{k+1}=A x_{k}+B w_{k}, w_{k} \in F,
$$

with a closed, convex bounded set $F \subset \mathbb{R}$. The state-independent bounds can be interpreted as a particular case of (3), with a constant limiting function $f(x)=\bar{w}$ which leads to $F=\{w:\|w\| \leq \bar{w}\}$.

In order to differentiate the robust positive invariance in the case of stateindependent bounds on disturbances (5) from the state-dependent counterpart (3)(4), the following definitions are introduced.

Definition 5. (F-invariance) A set $\Omega \subset \mathbb{R}^{n}$ is $F$-invariant with respect to (5) if $A x+B w \in \Omega$ for all $x \in \Omega$ and all $w \in F$.

Definition 6. The minimal F-invariant set is defined as the $F$-invariant set contained in any closed $F$-invariant set.

The minimal $F$-invariant set is unique, compact and contains the origin if $F$ contains the origin. Its $\epsilon$-neighborhood represents an ultimate bound for (5). It is well known $[8,15,17]$ that a Schur matrix $A$ leads to a minimal $F$-invariant set described as:

$$
M(F)=\lim _{k \rightarrow \infty} \bigoplus_{i=0}^{k} A^{i} B F .
$$

\subsection{Extension to state-dependent bounds}

The work in [17] concentrates principally on dynamic systems affected by bounded disturbances, but contains an extension to systems with bounded nonlinearity which gives a basic idea on the possible analysis of the state dependent disturbances in a set-theoretic framework. We reformulate here the main developments by adapting the construction in [17] to the present framework in order to analyse its mechanism and stress the working hypothesis.

Assumption 2 The state-dependent bound on the disturbances for system (3) is described by a function $f($.$) for which f\left(\mathbb{R}^{n}\right)=\left\{f(x): x \in \mathbb{R}^{n}\right\}$ is bounded. 
Under the Assumption 2 on the global bounds of the disturbance, one can initialize $M_{0}=\mathbb{R}^{n}$ and $F_{0}=\operatorname{Conv}\left\{f\left(M_{0}\right)\right\}$. By assuming $M_{j}$ to be $F_{j}$-invariant for $F_{j}=\operatorname{Conv}\left\{f\left(M_{j}\right)\right\}$ (which trivially holds for $M_{0}$ ) one can define the set sequence:

$$
M_{j+1}=M\left(F_{j}\right)
$$

as the minimal $F_{j}$-invariant for

$$
x_{k+1}=A x_{k}+B w_{k}, w_{k} \in F_{j} .
$$

Exploiting the inclusion $M_{j+1} \subset M_{j}$, one can conclude on the existence of convex sets defined as:

$$
M_{\infty}=\bigcap_{j=0}^{\infty} M_{j} \quad \text { and } F_{\infty}=\bigcap_{j=0}^{\infty} F_{j}
$$

Theorem 3. [17] Under the Assumptions 1 and 2, each of the sets $M_{j}, j=$ $0,1, \ldots, \infty$ is a robust positively invariant set for (3). If the initial state $x_{0}$ belongs to $M_{j}$ for some $j \in \mathbb{Z}_{+}$then the trajectory of the system (3) reaches in finite time an $\epsilon$-neighborhood of the set $M_{j+1}$ and remains in that neighborhood. Thus the system is ultimately bounded and the $\epsilon$-neighborhood of $M_{\infty}$ represents an ultimate bound.

The following remarks motivate our study.

Remark 1. The boundedness restrictions in Assumption 2 are relatively conservative. The procedure proposed in [17] cannot be initialized in the presence of radially unbounded functions ${ }^{3}$ describing the state-dependent limitations on the uncertainties (4) as long as $M_{0}$ will be unbounded.

Remark 2. Even in the case that Assumption 2 holds, Theorem 3 proposes a sequence of invariant sets but the asymptotic construction of $M_{\infty}$ leads to a limit-set (in fact its $\epsilon$-neighborhood) which represents a state-independent ultimate bound. This raises a question about the existence of a state-dependence (or parameterization) of the ultimate bounds with respect to the initial conditions.

There are simple examples to show that the above procedure can be refined towards state-dependent ultimate bounds. Consider the dynamics:

$$
x_{k+1}=0.5 x_{k}+w_{k}, \quad\left|w_{k}\right| \leq f\left(x_{k}\right)=\left|\frac{4 x_{k}^{2}-\left|x_{k}\right|}{4 x_{k}^{2}-1}\right|,
$$

\footnotetext{
${ }^{3}$ A function $f: \mathbb{R}^{n} \rightarrow \mathbb{R}$ is radially unbounded if $\|x\| \rightarrow \infty \Longrightarrow f(x) \rightarrow \infty$.
} 
where $f(x)$ is shown in Figure 1. Using the methodology in [17] that is described through (7)-(9), the nested set construction converges to $M_{\infty}=[-1.5 ; 1.5]$ and fails to identify all the intervals $[-c ; c]$ with $\frac{\sqrt{5}-2}{2} \leq c \leq 0.5$ which are robust positively invariant and represent tighter ultimate bounds if $x_{0} \in[-c ; c]$.

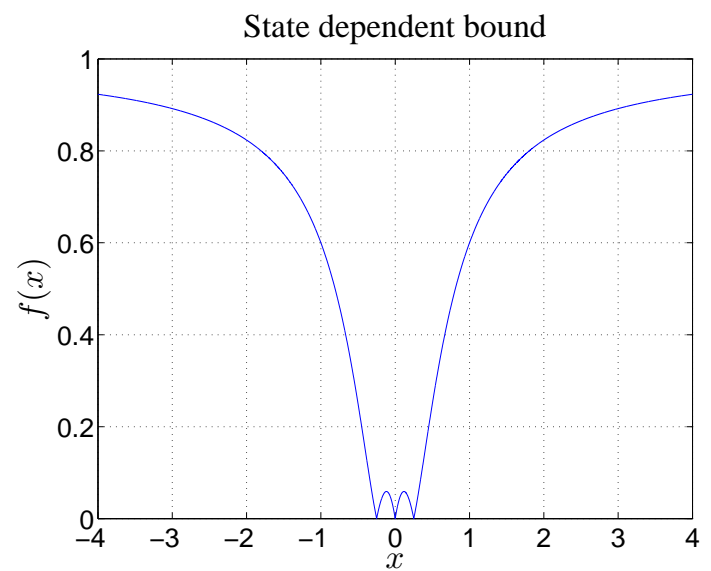

Fig. 1 Graphical description of the state dependent bound.

Figure 2 presents the time-domain simulations for different initial conditions illustrating the existence of invariant sets $[-1.5 ; 1.5]$ and $\left[-\frac{\sqrt{5}-2}{2} ; \frac{\sqrt{5}-2}{2}\right]$.
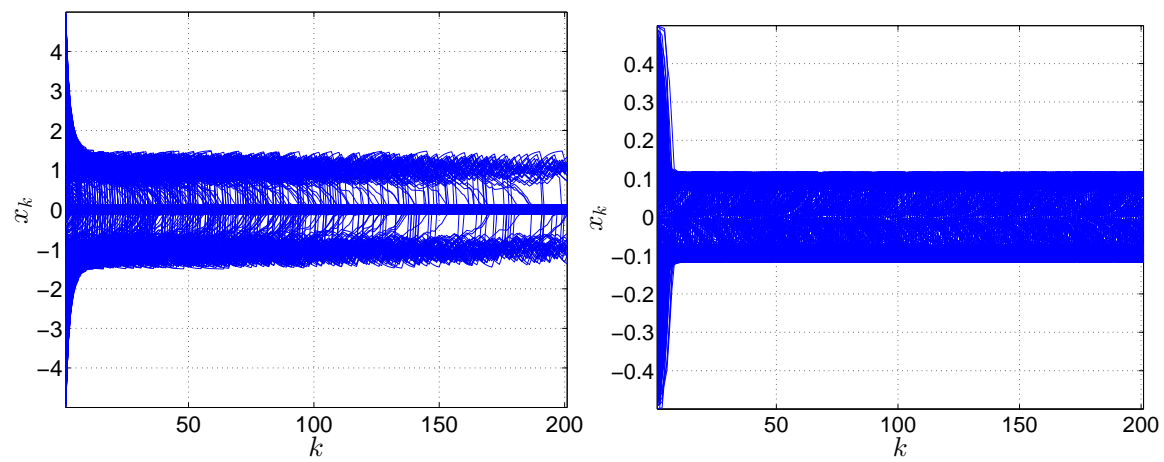

Fig. 2 Simulations with random initial conditions in $[-5 ; 5]$ - left; $[-0.5 ; 0.5]$ - right. 


\subsection{An existing result on ultimate bounds for systems with state dependent disturbances}

There exist a specific result [14] providing ultimate bounds expression for linear systems with state dependent perturbation bounds. The systematic method for their characterization under monotonicity conditions as underlined by the next theorem.

Theorem 4. [14] Consider the system (3), satisfying Assumption 1 with a Jordan canonical form of the transition matrix $A=V^{-1} \Lambda V$. Suppose that

$$
|B w| \leq g(|x|), \text { for all } x \in \mathbb{R}^{n}
$$

with a continuous map $g: \mathbb{R}_{+}^{n} \rightarrow \mathbb{R}_{+}^{n}$ verifying

$$
\left|y_{1}\right| \leq\left|y_{2}\right| \Longrightarrow g\left(\left|y_{1}\right|\right) \leq g\left(\left|y_{2}\right|\right)
$$

Consider the map:

$$
T(y) \triangleq|\Lambda| y+\left|V^{-1}\right| g(|V| y)
$$

and suppose that a point $b$ satsfying $b=T(b)$ exists. Let $y_{m} \in \mathbb{R}^{n}$ denote any point satisfying $\lim _{k \rightarrow \infty} T^{k}\left(\left|V^{-1} y_{m}\right|\right)=b$. If the initial condition $x_{0}$ satisfies $\left|V^{-1} x_{0}\right| \leq$ $\left|V^{-1} y_{m}\right|$ then for any $\epsilon \in \mathbb{R}_{+}^{n}$ there exists $l=l\left(\epsilon, y_{m}\right)$ such that for all $k \geq l$ :

$$
\begin{aligned}
\left|V^{-1} x_{k}\right| & \leq b+\epsilon \\
\left|x_{k}\right| & \leq|V| b+|V| \epsilon
\end{aligned}
$$

The result is remarkable in several respects. By building the ultimate bounds on the existence of fixed points for (13), the theorem opens the door for the characterization of different ultimate bounds (??) for the same dynamics. Each such ultimate bound has an associated domain of attraction identified via the collection of points $y_{m}$ in the statement. More than that, the ultimate bound (14) is robustly positive invariant. All these characteristics are providing suitable solutions with respect to the objectives of the present work. The assumptions are however relatively restrictive as detailed in the following remarks, thing which motivates the main results presented in the next section.

Remark 3. The monotonicity condition (12) is not fulfilled by the increasing functions from a particular point, as those described in Definition 2 which are related to star-shapedness and not with classical convexity of the level set of the bounding function. The monotonic functions are only a particular subclass of these increasing functions. 
Remark 4. The fixed-point condition for the map (13) builds on a series of overapproximations of the nonlinear state-dependent bounding function as for example those related to the component-wise absolute values of the matrices $V$ and $V^{-1}$. More than that, a qualitative analysis of the number of such fixed points and the correspondent basin of attraction is only implicitly embedded in the result. The settheoretic methods can offer alternative condition and describe parameters for the existence of ultimate bounds.

\section{Main result}

For a Schur matrix $A \in \mathbb{R}^{n \times n}$ and vector $B \in \mathbb{R}^{n}$ let us define the limit set

$$
\mathbb{M}=M\left(\mathbb{B}_{1}\right)=\lim _{k \rightarrow \infty} \bigoplus_{i=0}^{k} A^{i} B \mathbb{B}_{1},
$$

where $\mathbb{B}_{1}$ stands for the unit ball ${ }^{4}$. The set $\mathbb{M}$ is minimal robust positively invariant (RPI) set with respect to $x_{k+1}=A x_{k}+B w_{k} \forall w_{k} \in \mathbb{B}_{1}$ or equivalently for all $\left|w_{k}\right| \leq 1$.

In order to establish the main results we consider the Minkowski function $g_{\mathbb{M}}$ : $\mathbb{R}^{n} \rightarrow \mathbb{R}_{+}$associated to the set $\mathbb{M}$ :

$$
g_{\mathbb{M}}(x)=\inf \left\{\lambda \in \mathbb{R}_{+}: x \in \lambda \mathbb{M}\right\}
$$

Theorem 5. Let us consider the LTI system (3) with the state-dependent bound described by $f(x)$, an increasing function from $\bar{x}=0 \in \mathbb{R}^{n}$ in the sense of Definition 2. Consider the Minkowski function $g_{\mathbb{M}}(x)$ of the set $\mathbb{M}$ defined through (16)-(17), where the matrices $(A, B)$ characterize the system (3). The parameterized set

$$
\Omega_{\mathbb{M}}(\alpha)=\alpha \mathbb{M}
$$

is RPI with respect to (3) for any scalar positive parameters $\alpha$ in the set

$$
S_{\mathbb{M}}=\left\{\alpha \in \mathbb{R}_{+} \mid f(x) \leq g_{\mathbb{M}}(x) \forall x \in \mathcal{L}_{g_{\mathbb{M}}}(\alpha)\right\}
$$

where $\mathcal{L}_{g_{\mathbb{M}}}($.$) is the level set defined according to (1).$

\footnotetext{
${ }^{4}$ The unit ball is defined with respect to a predefined norm $|\cdot|_{p}$. In the present case the matrix $B \in \mathbb{R}^{n \times 1}$ and thus the corresponding unit ball is defined in $\mathbb{R}$ where the $|\cdot|_{p}$ are equivalent for $p \in[1, \infty)$.
} 
Proof: First note that $\Omega_{\mathbb{M}}(\alpha)$ is invariant with respect to $x_{k+1}=A x_{k}+$ $B w_{k}$, with $w_{k} \in \alpha \mathbb{B}_{1}$. Indeed using the definition (16) we have:

$$
\Omega_{\mathbb{M}}(\alpha)=\alpha \mathbb{M}=\alpha \lim _{k \rightarrow \infty} \bigoplus_{i=0}^{k} A^{i} B \mathbb{B}_{1}=\lim _{k \rightarrow \infty} \bigoplus_{i=0}^{k} A^{i} B\left\{\alpha \mathbb{B}_{1}\right\},
$$

which shows that $\Omega_{\mathbb{M}}(\alpha)$ is RPI.

Consider now a scalar $\alpha \in S_{\mathbb{M}}$. From the definition of the set $S_{\mathbb{M}}$ in (19):

$$
f\left(x_{1}\right) \leq g_{\mathbb{M}}\left(x_{1}\right)=\alpha \forall x_{1} \in \mathcal{L}_{g_{\mathbb{M}}}(\alpha)
$$

The fact that $f(x)$ is an increasing function from $0 \in \mathbb{R}^{n}$ ensures on one hand that $\mathcal{L}_{f}^{-}\left(c_{1}\right) \supseteq \mathcal{L}_{f}^{-}\left(c_{2}\right) \supset\{0\}$ if $c_{1} \geq c_{2}>0$, and on the other the star-shape property of the sublevel set of $f($.$) . Exploiting this last property, for any x_{2} \in \Omega_{\mathbb{M}}(\alpha)$ there exists a scalar $0 \leq \beta \leq 1$ such that $x_{2}=\beta x_{1}$ and $x_{1} \in \mathcal{L}_{g_{\mathbb{M}}}(\alpha)$. From the definition of the sublevel set (1) we have:

$$
f\left(x_{2}\right) \leq f\left(x_{1}\right)
$$

From (21) and (22) it yields $f\left(x_{2}\right) \leq \alpha$ and subsequently:

$$
\left|w_{k}\right| \leq \alpha \forall x_{k} \in \alpha \mathbb{M}=\Omega_{\mathbb{M}}(\alpha)
$$

In this way, the proof of invariance of $\Omega_{\mathbb{M}}(\alpha)$ is completed.

The problems formulated in Subsection 2.1 can be addressed in light of the Theorem 5. The next Corollaries resume these basic sufficient conditions for the existence of ultimate bounds represented by convex sets and their parametrization with respect to the initial conditions.

Corollary 1. The solution $x_{k}=x\left(x_{0}, \mathrm{w}^{0: k-1}\right)$ of (3) is globally ultimately bounded if the set $S_{\mathbb{M}}$ in (19) exists and is unbounded. Additionally, if $S_{\mathbb{M}}=\mathbb{R}_{+}$then the origin is a robustly asymptotically stable equilibrium point.

Proof: If the set $S_{\mathbb{M}}$ is unbounded, then there exists a subset $[\bar{c}, \infty) \subseteq S$ such that $\Omega_{\mathbb{M}}(\alpha)$ is RPI for all $\alpha \in[\bar{c}, \infty)$. But these sets are also attractive (in the virtue of the properties of the minimal RPI sets with constant bounds) and by consequence $\Omega_{\mathbb{M}}(\bar{c})$ will represent a global ultimate bound for the state trajectories. For the second part of the Corollary, it is easy to observe that $\bar{c}=0$ and thus any neighborhood of the origin $[-\epsilon, \epsilon]$ can be reached in a finite number of iterations independently of the initial conditions. It follows that $x_{k} \rightarrow 0$ as $k \rightarrow \infty$.

The Corollary 1 offers a sufficient condition for global ultimate boundedness and robust asymptotic stability in the presence of state dependent disturbances. It is 
worth to mention that this condition admits state dependent bounds described by radially unbounded functions $f($.$) (which is not the case of Theorem 3). Indeed, the$ only condition to be satisfied in Theorem 5 is $f(x)<g_{\mathbb{M}}(x), \forall x \in \mathbb{R}^{n}$ and, by definition, the function $g_{\mathbb{M}}($.$) is radially unbounded.$

Corollary 2. Consider the system (3) under the assumptions of Theorem 5 and the set $S_{\mathbb{M}} \subseteq \mathbb{R}_{+}$in (19). If $S_{\mathbb{M}}$ is unbounded and described by a (possibly infinite) union of disjoint intervals:

$$
S_{\mathbb{M}}=\left[\underline{c}_{1}, \bar{c}_{1}\right) \cup\left[\underline{c}_{2}, \bar{c}_{2}\right) \cup \cdots \cup\left[\underline{c}_{i}, \bar{c}_{i}\right) \ldots
$$

with $0=\bar{c}_{0} \leq \underline{c}_{1}<\bar{c}_{1}<\underline{c}_{2}<\bar{c}_{2}<\ldots \underline{c}_{i}<\bar{c}_{i}<\ldots$ then $\Omega_{\mathbb{M}}\left((1+\epsilon) \underline{c}_{i+1}\right)$ is an ultimate bound for $\epsilon \in\left(0, \frac{\bar{c}_{i+1}-\underline{c}_{i+1}}{\underline{c}_{i+1}}\right)$ and any $x_{0} \in \Omega_{\mathbb{M}}(c)$ with $c \in\left[\bar{c}_{i}, \bar{c}_{i+1}\right)$.

Proof: We split the interval $\left[\bar{c}_{i}, \bar{c}_{i+1}\right)=\left[\bar{c}_{i}, \underline{c}_{i+1}\right) \cup\left[\underline{c}_{i+1}, \bar{c}_{i+1}\right)$. On one hand, for any initial condition in $\Omega_{\mathbb{M}}(\underline{c})$ with $c \in\left[\underline{c}_{i+1}, \bar{c}_{i+1}\right)$, an ultimate bound can be obtained using the $\epsilon$-outer approximation of $\Omega_{\mathbb{M}}\left(\underline{c}_{i+1}\right)$ with a similar argument used in Corollary 1. On the other hand, it can be observed that $x_{0} \in \Omega(c) \subset \Omega\left(\underline{c}_{i+1}\right)$ for any $c \in\left[\bar{c}_{i}, \underline{c}_{i+1}\right)$ and the ultimate boundedness of the trajectories follows from the robust positive invariance of of $\Omega\left(\underline{c}_{i+1}\right)$.

Example 1: Consider the dynamical system:

$$
x_{k+1}=0.5 x_{k}+w_{k},\left|w_{k}\right| \leq f\left(x_{k}\right)=\left|x_{k}\right|^{\frac{1}{2}} .
$$

Figure 3 shows the relationship between $f(x)$ and $g_{\mathbb{M}}(x)$. The last one represents the Minkowski function corresponding to the minimal invariant set $\mathbb{M}=[-2,2]$ in (16). Note that the function $f(x)$ is radially unbounded and thus Theorem 3 cannot be applied. Using Theorem 5 one can describe the set of admissible parameters $S_{\mathbb{M}}=$ $[2, \infty)$ leading to admissible invariant sets $\Omega_{\mathbb{M}}(\alpha)=\alpha[-2 ; 2] \forall \alpha \in S_{\mathbb{M}}$ and the global ultimate bound $\Omega_{\mathbb{M}}((1+\epsilon) 2)=2(1+\epsilon)[-2 ; 2]=(1+\epsilon)[-4 ; 4], \epsilon>0$. Note also, in the virtue of the Corollary 1 , that the origin is not a robust asymptotically stable equilibrium point.

The construction of the set $S_{\mathbb{M}}$ in Theorem 5 might be seen as a difficult task as long as it involves the Minkowski function of minimal robust positive invariant set $\mathbb{M}$ in (6). It is known that $\mathbb{M}$ being the limit set of an infinite Minkowsi sum has a finite (explicit) representation in terms of generators only for restricted classes of LTI dynamics (in the case $A^{k} B=\alpha B$ for some $k \in \mathbb{N}_{+}$and $0 \leq \alpha \leq 1$ ). As such, for practical reasons, the use of approximations are enabled along the lines of the next results. 

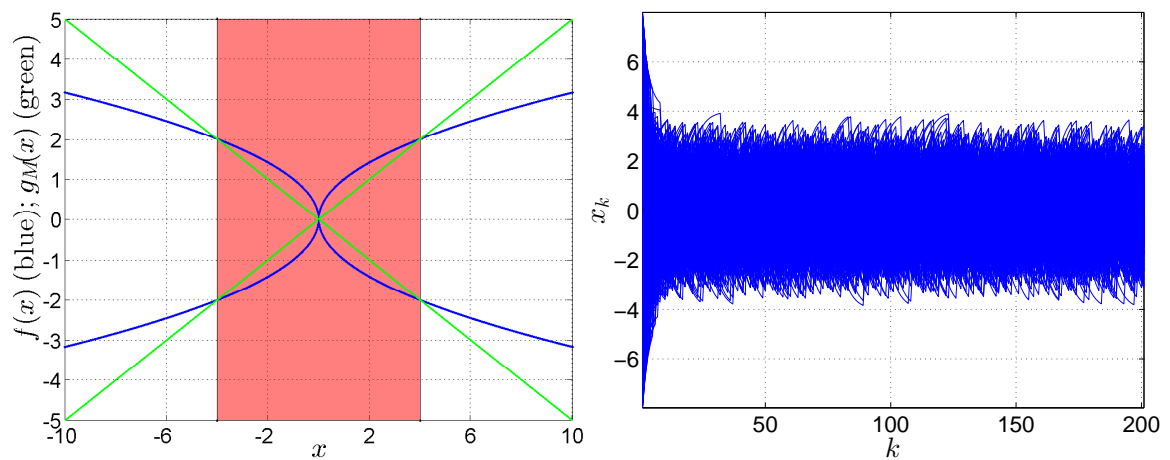

Fig. 3 Left: the graph of $f(x)$ (blue) and $g_{\mathbb{M}}(x)$ (green) - describing the envelope (positive and negative) bounds of the disturbances. The red interval represents the region for which $f(x)>$ $g_{\mathbb{M}}(x)$ and thus correspond to scaling factors $\alpha \notin S$. Right: time simulations with random initial conditions in $[-8,8]$.

Theorem 6. Let $\mathbb{U}$ be a polyhedral RPI set with respect to $x_{k+1}=A x_{k}+B w_{k}$ with $w_{k} \in \mathbb{B}_{1}$. The parameterized set $\Omega_{\mathbb{U}}(\alpha)=\alpha \mathbb{U}$ is $R P I$ with respect to (3) for all $\alpha$ in:

$$
S_{\mathbb{U}}=\left\{\alpha \in \mathbb{R}_{+} \mid f(x) \leq g_{\mathbb{U}}(x) \forall x \in \mathcal{L}_{g_{\mathbb{U}}}(\alpha)\right\}
$$

The proof is similar to the one in Theorem 5 and is omitted.

Example 2: Consider the two dimensional dynamical system:

$$
\left[\begin{array}{l}
x_{1, k+1} \\
x_{2, k+1}
\end{array}\right]=\left[\begin{array}{cc}
0.2 & 0.2 \\
0 & 0.4
\end{array}\right]\left[\begin{array}{l}
x_{1, k} \\
x_{2, k}
\end{array}\right]+\left[\begin{array}{l}
0 \\
1
\end{array}\right] w_{k}
$$

where $\left|w_{k}\right| \leq f\left(x_{k}\right)=\left|x_{1, k}\right|+0.1\left|x_{1, k}\right|^{0.2}\left|x_{2, k}\right|^{0.2}+\left|x_{2, k}\right|^{0.5}$ and $f($.$) fulfills the$ increasing from 0 assumption as illustrated in Figure 4 . The minimal invariant set $\mathbb{M}$ in this case will be replaced by a tight outer invariant approximation $\mathbb{U}$, illustrated in Figure 4 with its level sets ${ }^{5}$. The superposition of the functions $f(x)$ and $g_{\mathbb{U}}(x)$ is given in Figure 5 where it can be seen that their intersection is done along nonconvex curves. The right hand side of the same figure, shows the regions for which $f(x) \geq g_{\mathbb{U}}(x)$ together with a value of the scalar $\alpha$ such that the parameterized set $\Omega_{\mathbb{U}}(\alpha)$ is guaranteed to be RPI. This provides an exemplification of the analysis tools available via Theorem 5 .

\footnotetext{
${ }^{5}$ This particular function is increasing from $\bar{x}=0$ in the sense of Definition 2 but not monotonic according to (12) and thus the hypothesis of Theorem 4 are not satisfied in this case.
} 

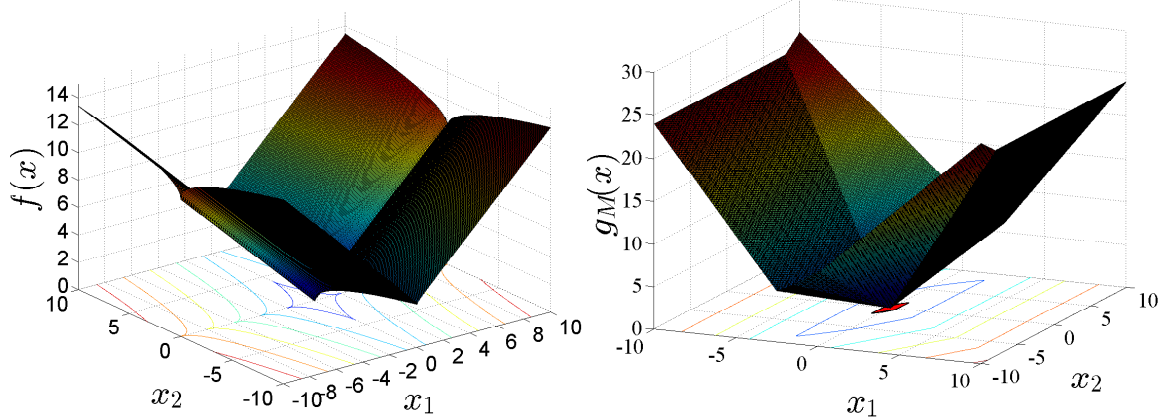

Fig. 4 Left: the graph of $f(x)$. Right: the graph of $g_{\mathbb{U}}(x)$.
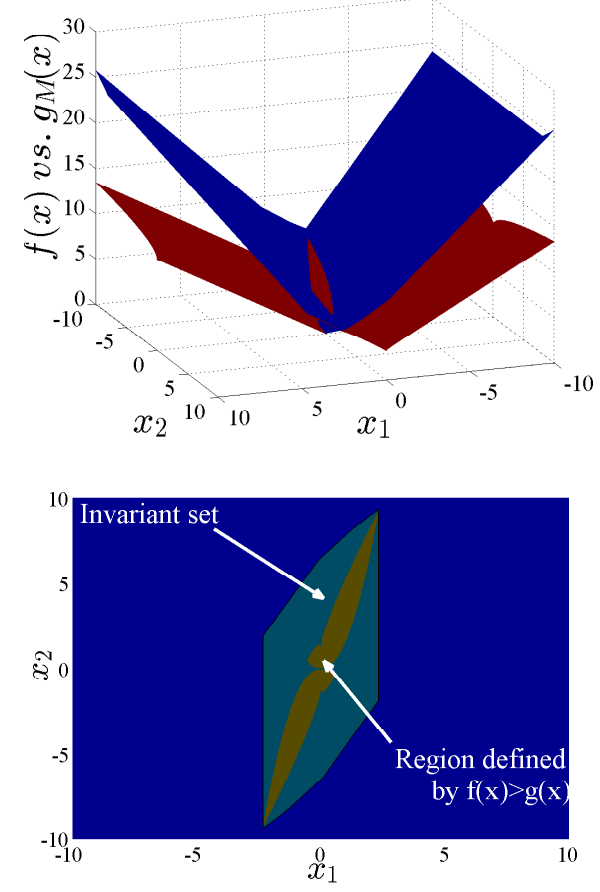

Fig. 5 Top: the graph of $f(x)$ (red) and $g_{\mathbb{M}}(x)$ (green). Bottom: 2D illustration of the shape of the RPI set in comparison with the region for which $f(x) \geq g_{\mathbb{U}}(x)$.

Remark 5. For constructing the parameterized set $\Omega_{\mathbb{U}}(\alpha)$ one can use low complexity invariant approximation $[14,21,22]$ of the set $\mathbb{M}$ in $(6)$, as for example:

$$
\mathbb{U}=\left\{x:\left|V^{-1} x\right| \leq(I-|\Lambda|)^{-1}\left|V^{-1}\right||B|\right\}
$$


with $\Lambda=V^{-1} A V$, corresponding to the Jordan canonical form of the transition matrix in (3). The function $g_{\mathbb{U}}($.$) corresponds to a polyhedral cone and is piecewise$ linear over a cone-partition of the states space.

Proposition 1. Let $\mathbb{M}$ be the minimal RPI set with respect to $x_{k+1}=A x_{k}+B w_{k}$ with $w_{k} \in \mathbb{B}_{1}$. If $\mathbb{U}$ is a polyhedral RPI approximation of $\mathbb{M}$ then $S_{\mathbb{M}} \supseteq S_{\mathbb{U}}$, where $S_{\mathbb{M}}$ and $S_{\mathbb{U}}$ are constructed based on (19) and (26) for a given function $f($.$) increas-$ ing from 0 .

Proof: Note that $\mathbb{U} \supseteq \mathbb{M}$ by the properties of the minimal RPI set. This fact implies $g_{\mathbb{U}}(x) \leq g_{\mathbb{M}}(x) \forall x \in \mathbb{R}^{n}$ and this relationship can be related to the inequalities involved in (19)-(26) where $f(x) \leq g_{\mathbb{U}}(x)$ only if $f(x) \leq g_{\mathbb{M}}(x)$. Under the starshaped assumption for $f(x)$ it follows that $S_{\mathbb{M}} \supseteq S_{\mathbb{U}}$ and thus the approximation will be inherited by the parameterized set of RPI sets for which the sufficient conditions hold.

An illustration of the impact of the invariant set approximation on the function entering in the comparison with the state-dependent bound in Theorem 5 in given in Figure 6.

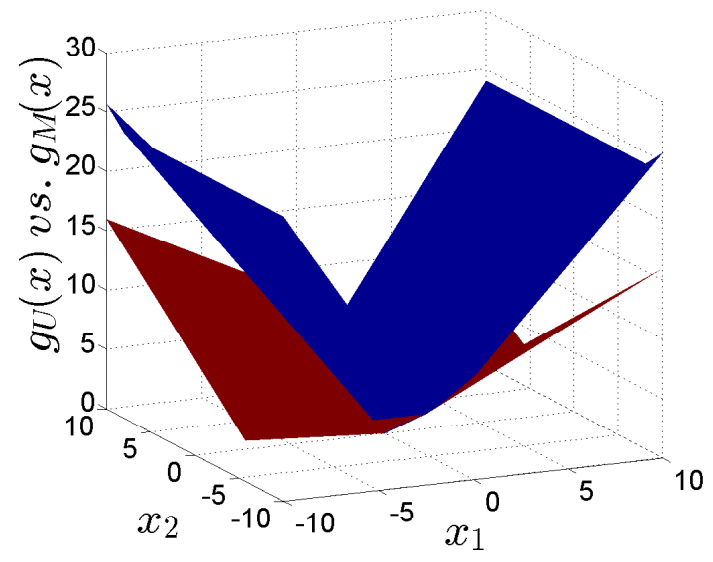

Fig. 6 The comparision of a tight approximation of the graph of $g_{\mathbb{M}}(x)$ (blue) based on the tight approximation of the minimal RPI set in (6) and $g_{\mathbb{U}}(x)$ (red) based on (28) for the LTI system in Example 2.

Remark 6. Theorem 5 builds on the assumption of a bounding function increasing from 0 which is satisfied for the Examples 1 and 2. However, the system in (10) 
violates this assumption which is based on the star-shape property of the sublevel set. Indeed, a simple check shows that

$$
\mathcal{L}_{f}^{-}(0.05)=[-0.071 ; 0.071] \cup[0.167 ; 0.306] \cup[-0.306 ;-0.167]
$$

Another example of bounding function which doesn't satisfy the increasing assumption will be the Himmelblau's function for the bound of the disturbance with respect the system dynamics in (27). This function is presented in Figure 7 with the corresponding contour (level sets) which are non-connected and cannot lead to RPI sets centered in the origin.
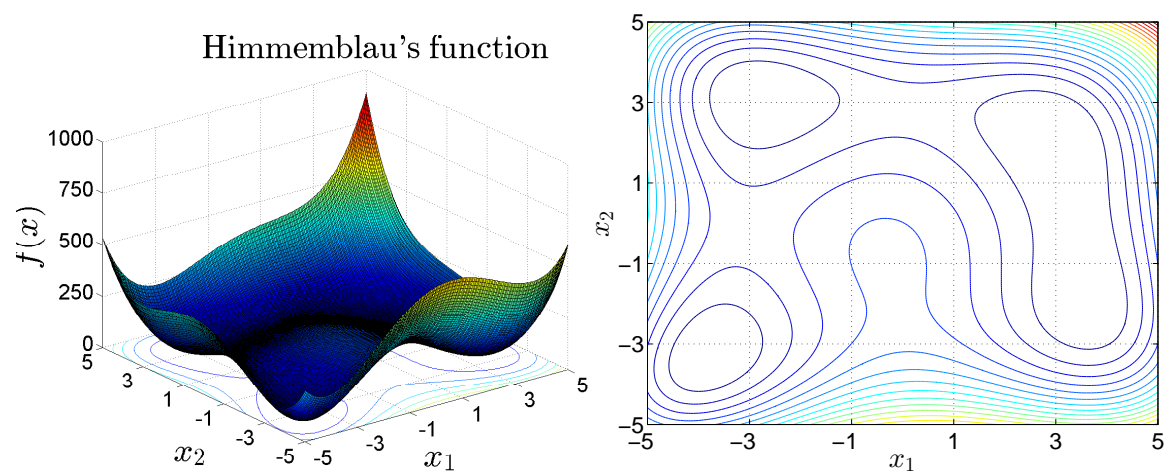

Fig. 7 Left: the graph of Himmelblau's function $f\left(\left[x_{1} x_{2}\right]^{T}\right)=\left(x_{1}^{2}+x_{2}-11\right)^{2}+\left(x_{1}+x_{2}^{2}-7\right)^{2}$. Right: the corresponding (non-connected) level sets.

In order to apply Theorem 5 for any state-dependent bound on the disturbance (see Remark 6), embedding via a star-shaped envelope can be used as follows:

Proposition 2. Let $f: \mathbb{R}^{n} \rightarrow \mathbb{R}_{+}$and a point $\bar{x} \in \mathbb{R}^{n}$. The function $h: \mathbb{R}^{n} \rightarrow \mathbb{R}_{+}$ defined as

$$
h(x)=\max _{0 \leq \gamma \leq 1} f(\gamma x+(1-\gamma) \bar{x})
$$

is increasing from $\bar{x}$ and $f(x) \leq h(x) \forall x \in \mathbb{R}^{n}$.

Proof: Direct application of the star-shape properties in Definitions 1-2.

For the system in (10), the use of the star-shape embedding (Figure 8) lead to the identification of the set of admissible parameters $S_{\mathbb{M}}=\left[\frac{\sqrt{5}-2}{4}, 0.25\right) \cup[0.75, \infty)$ for the parameterized invariant sets $\Omega_{\mathbb{M}}=\alpha[-2,2], \alpha \in S_{\mathbb{M}}$ illustrated by the comparison between $h(x)$ and $g_{\mathbb{M}}(x)$ in Figure 9. 


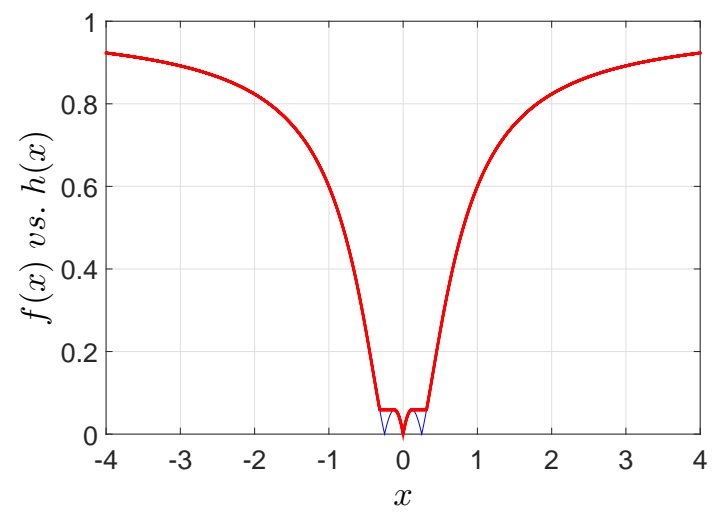

Fig. $8 f(x)$ (blue) in (10) and its star-shaped embedding $h(x)$ (red).

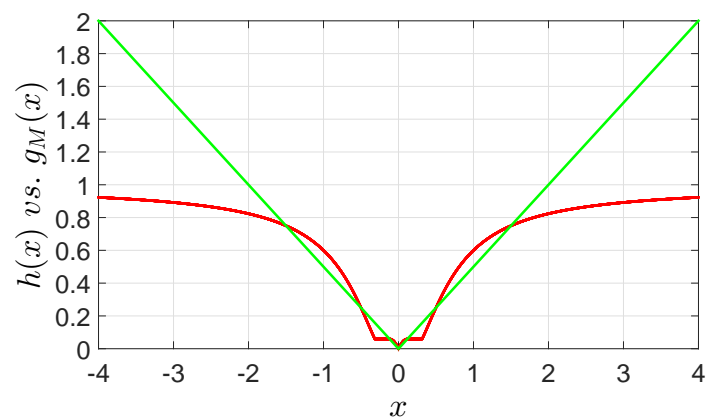

Fig. $9 h(x)$ (red) compared to the Minkowski function $g_{\mathbb{M}}(x)$ (green).

\section{Extensions and connections with optimization-based design}

\subsection{Extensions}

Based on the main results of this study presented in Section 3, it is worth to determine the parameterized sets $\Omega_{\mathbb{M}}(\alpha)$ for special cases of the bound-function $f(x)$ summarized in the following corollaries.

Corollary 3. Consider system (3). If $f(x)$ is convex then $\Omega_{\mathbb{M}}(\alpha)=\alpha \mathbb{M}$ is an invariant set for all $\alpha_{m} \leq \alpha \leq \alpha_{M}$ with:

$$
\alpha_{m}=\inf \left\{\alpha \in \mathbb{R}_{+} \mid f(x) \leq g_{\mathbb{M}}(x) \forall x \in \mathcal{L}_{g_{\mathbb{M}}}(\alpha)\right\}
$$




$$
\alpha_{M}=\sup \left\{\alpha \in \mathbb{R}_{+} \mid f(x) \leq g_{\mathbb{M}}(x) \forall x \in \mathcal{L}_{g_{\mathbb{M}}}(\alpha)\right\}
$$

Definition 7. The nonlinear function $f: \mathbb{R}^{n} \rightarrow \mathbb{R}_{+}$is continuous and conebounded over $\mathbb{R}^{n}$, if there exist non-negative constants $\lambda_{0}$ and $\lambda_{1}$ such that

$$
\|f(x)\| \leq \lambda_{0}+\lambda_{1}\|x\| \forall x \in \mathbb{R}^{n} .
$$

Corollary 4. Consider the system (3) where the additive uncertainties satisfy (4) with a cone-bounded function $f($.$) as described in Definition 7. The parameterized$ set $\Omega_{\mathbb{M}}(\alpha)=\alpha \mathbb{M}$ is robustly invariant for all $\alpha \geq \alpha_{m}$ with $\alpha_{m}$ given by (30).

Remark 7. In [16] several classes of uncertain nonlinear dynamics have been mentioned in the context of state dependent uncertainties, as for example:

$$
x_{k+1}=A x_{k}+c_{k} g\left(x_{k}\right)+z_{k},
$$

with $c_{k}, z_{k} \in \mathbb{R}^{n}$ satisfying elementwise the inequality $\left\|c_{k}\right\| \leq C_{\max },\left\|z_{k}\right\| \leq$ $Z_{\max }$ with $C_{\max }, Z_{\max } \in \mathbb{R}_{+}^{n}$, and a scalar function of a vector argument $g$ : $\mathbb{R}^{n} \rightarrow \mathbb{R}_{+}$. The system (33) is an example of dynamics which can be regarded as a linear subject to a cone-bounded uncertainty and parameterized sets $\Omega_{\mathbb{M}}(\alpha)$ can be determined according to Corollary 4.

Corollary 5. Consider the system

$$
x_{k+1}=A x_{k}+B w_{k}+B_{u} \bar{u},\left|w_{k}\right| \leq f\left(x_{k}\right),
$$

satisfying Assumption 1, with $f($.$) a increasing function from (I-A)^{-1} B_{u} \bar{u}$, $B_{u} \in \mathbb{R}^{n}$ and a constant signal $\bar{u}$. The parameterized set $\Omega_{\mathbb{M}}(\alpha, \bar{u})=\alpha \mathbb{M} \oplus$ $(I-A)^{-1} B_{u} \bar{u}$ is robustly invariant for all $\alpha \in \mathcal{S}_{\mathbb{M}}(\bar{u})$ with $\mathcal{S}_{\mathbb{M}}(\bar{u})$ computed based on Theorem 5 for the system $\xi_{k+1}=A \xi_{k}+B w_{k}$ subject to constraints $\left|w_{k}\right| \leq f\left(\xi_{k}+(I-A)^{-1} B_{u} \bar{u}\right)$.

Proof: We observe that in the absence of disturbances, the trajectories converge to $\bar{x}=(I-A)^{-1} B_{u} \bar{u}$. Then the analysis can be done with respect to the shifted dynamics $x_{k}=\bar{x}+\xi_{k}$ with the particularity that the function $g_{\mathbb{M}}\left(\xi_{k}\right)$ is star-shaped in 0 while the original bounding function $f($.$) is described in the original state space$ and thus it is computed according to the change of variable $f\left(\xi_{k}+\bar{x}\right)$. 


\subsection{Detection of mode switching via set invariance}

The positive invariance of a set with respect to the nominal dynamics is a strong notion and can be exploited for the detection of a switch in the dynamics [28]. The basic idea is to construct off-line the family of invariant sets and monitor in real time the inclusion of the state in the respective set. In case that the invariance is violated, then a change of mode is detected. Subsequently, the convergence to a different invariant (limit set) can lead to the identification of the current mode of functioning. This mechanism has been documented and is well understood for linear dynamical systems in the presence of bounded disturbances. We will prove in the next paragraphs the way the theoretical developments on the state-dependent ultimate bounds can be used in practice.

The off-line construction of the family of invariant sets is realized based on the following proposition (the proof is omitted for brevity).

Proposition 3. Let us consider a dynamical system described by

$$
x_{k+1}=A_{i} x_{k}+B_{i} w_{k}+B_{u i} \bar{u}_{i},\left|w_{k}\right| \leq f_{i}\left(x_{k}\right)
$$

with $i_{k} \in\{1,2\}$ a switching signal in between two modes. It is considered that for each mode we can construct independently the parameterized invariant sets $\Omega_{\mathbb{M}}^{i}(\alpha, \bar{u}), \alpha \in \mathcal{S}_{\mathbb{M}}^{i}(\bar{u})$ according to Corollary 5 .

- If $x_{k} \in \Omega_{\mathbb{M}}^{i}(\alpha, \bar{u})$, where $\Omega_{\mathbb{M}}^{i}$ is RPI with respect to the $i$-th mode of the dynamics of system (29) with $i \in\{1,2\}, \alpha \in \mathcal{S}_{\mathbb{M}}^{i}$ and $x_{k+1} \notin \Omega_{\mathbb{M}}^{i}(\alpha, \bar{u})$ then a switch took place.

- Consider additionally that

$$
S_{\mathbb{M}}^{i}(\bar{u})=\left[\underline{c}_{1}^{i}, \bar{c}_{1}^{i}\right) \cup\left[\underline{c}_{2}^{i}, \bar{c}_{2}^{i}\right) \cup \cdots \cup\left[\underline{c}_{j}^{i}, \bar{c}_{j}^{i}\right) \ldots ; i \in\{1,2\},
$$

and $x_{0} \in \Omega_{\mathbb{M}}^{1}\left(\alpha^{1}, \bar{u}\right) \cap \Omega_{\mathbb{M}}^{2}\left(\alpha^{2}, \bar{u}\right)$ for $\alpha^{1} \in\left[\bar{c}_{j}^{1}, \bar{c}_{j+1}^{1}\right) \subseteq \mathcal{S}_{\mathbb{M}}^{1}(\bar{u}), \alpha^{2} \in$ $\left[\bar{c}_{l}^{2}, \bar{c}_{l+1}^{2}\right) \subseteq \mathcal{S}_{\mathbb{M}}^{2}(\bar{u})$. The time-invariant mode of functioning can be identified if $\Omega_{\mathbb{M}}^{1}\left(\underline{c}_{j+1}^{1}, \bar{u}\right) \cap \Omega_{\mathbb{M}}^{2}\left(\underline{c}_{l+1}^{2}, \bar{u}\right)=\emptyset$.

A simple way to exploit the result is to consider the auxiliary signal $\bar{u}$ as a degree of freedom for separation of the ultimate bounds for the modes of functioning in a switching dynamical system. The basic idea is to find the auxiliary signal $\bar{u}$, minimum in norm such that the ultimate bounds corresponding to a give state are separated. Taking into account Proposition 3, we formulate for $x \in \mathbb{R}^{n}$ the following optimization problem in a compact form: 


$$
\begin{aligned}
\min _{\bar{u}} & |\bar{u}| \\
\text { subject to: } & \Omega_{\mathbb{M}}^{1}\left(\underline{c}_{j+1}^{1}, \bar{u}\right) \cap \Omega_{\mathbb{M}}^{2}\left(\underline{c}_{l+1}^{2}, \bar{u}\right)=\emptyset
\end{aligned}
$$

where $j$ and $l$ are such that $x \in \Omega_{\mathbb{M}}^{1}\left(\alpha^{1}, \bar{u}\right) \cap \Omega_{\mathbb{M}}^{2}\left(\alpha^{2}, \bar{u}\right)$. The optimization is nonlinear and highly correlated with the state-dependant bounds.

If $S_{\mathbb{M}}^{i}$ are non-connected sets then each sub-interval should be treated independently. Note however that the obtained ultimate bounds for each interval of parameters are convex sets and that for specific classes of state dependent bounds (convex, cone-bounded) $S_{\mathbb{M}}^{i}$ is a connected set, see Corollary 3-4.

Example: Consider the dynamical system (35) with:

$$
\begin{gathered}
A_{1}=\left[\begin{array}{cc}
1 & 0.1 \\
-0.9 & 0
\end{array}\right] ; B_{1}=\left[\begin{array}{l}
0 \\
1
\end{array}\right] ; B_{1 u}=\left[\begin{array}{l}
0 \\
0
\end{array}\right] ; \\
A_{2}=\frac{1}{3}\left[\begin{array}{cc}
1 & -0.2 \\
-0.2 & 0.5
\end{array}\right] ; B_{2}=\left[\begin{array}{l}
1 \\
1
\end{array}\right] ; B_{2 u}=\left[\begin{array}{l}
1 \\
0
\end{array}\right] ; \\
f_{1}\left(x_{k}\right)=0.1+\left|0.7 * \sin \left(x_{k, 1}\right)-x_{k, 1}\right| ; f_{2}\left(x_{k}\right)=1 .
\end{gathered}
$$

The dynamics of the first mode is not affected by the exogenous signal $\bar{u}$ and as such the parameterized family of ultimate bounds (Figure 10) will be described by the union of intervals:

$$
\begin{aligned}
S_{\mathbb{U}}^{1}(\bar{u})= & {[1.1 ; 2.2) \cup[5 ; 6.8) \cup[8.9 ; 11.5) \cup[13.8 ; 16.1) \cup[18.5 ; 20.8) } \\
& \cup[22.8 ; 25.5) \cup[26.8, \ldots
\end{aligned}
$$

The second mode of functioning is linear and the parameterized invariant set is given by:

$$
\Omega_{\mathbb{M}}^{2}(\alpha, \bar{u})=\alpha \mathbb{M} \oplus(I-A)^{-1} B_{u} \bar{u}
$$

with $\alpha \in[1, \infty)$ and $\bar{u} \in \mathbb{R}$.

Solving 4 Linear Programming problems for each of the intervals in (40) one can find the level of $\bar{u}$ which ensures asymptotic mode detection - Figure 11.

\section{Conclusion and further research directions}

In this paper we revisit the ultimate bounds for linear systems in presence of additive disturbances. We extend their characterization from the classical case of fix bounds 


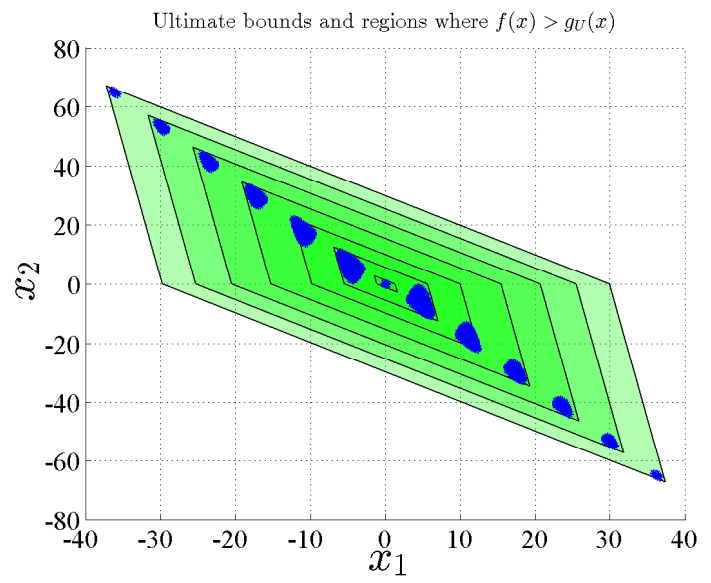

Parameterized invariant set as a function of $\alpha$

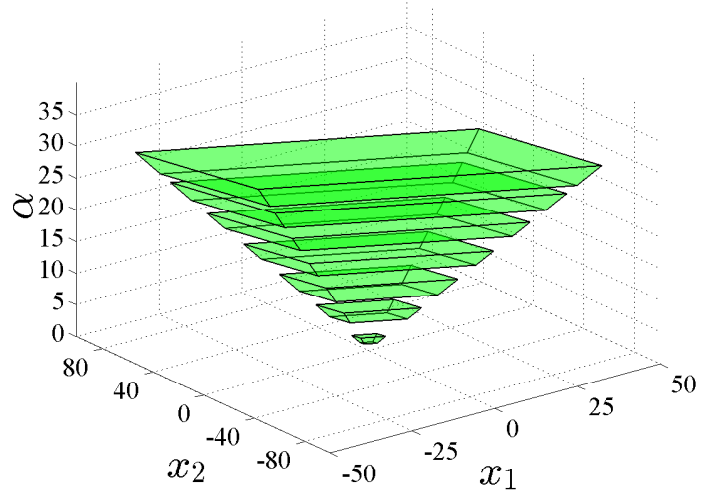

Fig. 10 The ultimate bounds $\Omega_{\mathbb{M}}^{1}(\alpha, \bar{u})$ obtained by exploiting the relationship between the state dependent noise-bounding function $f(x)$ and the function $g_{\mathbb{U}}(x)$ on the left. The interval of scaling factors $\alpha$ corresponding to invariant sets $\Omega_{\mathbb{M}}^{1}(\alpha)$ - right.

to the state-dependent bounds. It is shown that a particular function can be defined over the state space and serve as a comparison for the state-dependent bounds.

In the case of multiple sources of additive disturbance, the present study can be extended to account for element-wise state dependent bounds by analyzing independently each column of the matrix $B \in \mathbb{R}^{n \times m}$. 

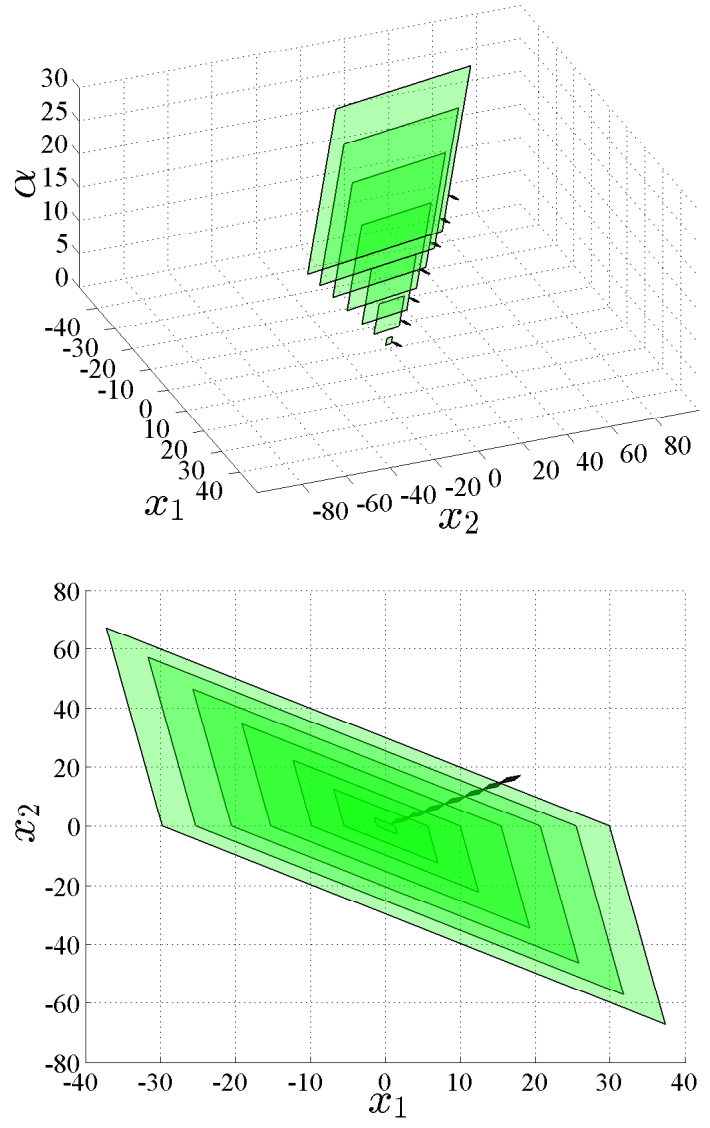

Fig. 11 The separation of ultimate bounds for different intervals of scaling coefficients $\Omega_{\mathbb{M}}^{1}\left(\underline{c}_{j+1}^{1}\right) \cap \Omega_{\mathbb{M}}^{2}(1, \bar{u})=\emptyset$.

\section{Acknowledgment}

The results presented in this chapter were initiated in the framework of the FrenchItalian collaborative research project Galileo 2014. The authors would like to thank Prof. F. Blanchini, Prof. D. Casagrane, Prof. S. Miani and G. Giordano for the fruitful discussion on the topic. This work was supported by the People Programme (Marie Curie Actions) of the European FP7 (2007-2013) under REA grant agreement $n^{\circ} 626891$. 


\section{References}

1. J.-P. Aubin. Viability theory. Springer Science \& Business Media, 2009.

2. J.-P. Aubin and H. Frankowska. Set-valued analysis. Springer, 2009.

3. B. R. Barmish and J. Sankaran. The propagation of parametric uncertainty via polytopes. IEEE Transactions on Automatic Control, 24(2):346-349, 1979.

4. B.R. Barmish and G. Leitmann. On ultimate boundedness control of uncertain systems in the absence of matching assumptions. IEEE Transactions on Automatic Control, 27(1):153-158, 1982.

5. D. P. Bertsekas and I. B. Rhodes. Recursive state estimation for a set-membership description of uncertainty. IEEE Transactions on Automatic Control, 16(2):117-128, 1971.

6. G. Bitsoris. Positively invariant polyhedral sets of discrete-time linear systems. International Journal of Control, 47(6):1713-1726, 1988.

7. F. Blanchini. Ultimate boundedness control for uncertain discrete-time systems via setinduced lyapunov functions. In Proceedings of the 30th IEEE Conference on Decision and Control, pages 1755-1760, 1991.

8. F. Blanchini and S. Miani. Set-theoretic methods in control. Springer, 2007.

9. E. De Santis. On positively invariant sets for discrete-time linear systems with disturbance: an application of maximal disturbance sets. IEEE Transactions on Automatic Control, 39(1):245249, 1994.

10. E. De Santis. Invariant sets: A generalization to constrained systems with state dependent disturbances. In Proceedings of the 37th IEEE Conference on Decision and Control, volume 1, pages 622-623, 1998.

11. X. Feng, V. Puig, C. Ocampo-Martinez, S. Olaru, and F. Stoican. Set-theoretic methods in robust detection and isolation of sensor faults. International Journal of Systems Science, 46(13):2317-2334, 2015.

12. J. D. Glover and F. C. Schweppe. Control of linear dynamic systems with set constrained disturbances. IEEE Transactions on Automatic Control, 16(5):411-423, 1971.

13. E. C. Kerrigan. Robust constraint satisfaction: Invariant sets and predictive control. $\mathrm{PhD}$ thesis, University of Cambridge, 2001.

14. E. Kofman, H. Haimovich, and M. M. Seron. A systematic method to obtain ultimate bounds for perturbed systems. International Journal of Control, 80(2):167-178, 2007.

15. I. Kolmanovsky and E. G. Gilbert. Theory and computation of disturbance invariant sets for discrete-time linear systems. Mathematical Problems in Engineering, 4(4):317-367, 1998.

16. A.V. Kuntsevich and V. M. Kuntsevich. Invariant sets for families of linear and nonlinear discrete systems with bounded disturbances. Automation and Remote Control, 73(1):83-96, 2012.

17. V. M. Kuntsevich and B. N. Pshenichnyi. Minimal invariant sets of dynamic systems with bounded disturbances. Cybernetics and Systems Analysis, 32(1):58-64, 1996.

18. G. Leitmann. Guaranteed asymptotic stability for a class of uncertain linear dynamical systems. Journal of Optimization Theory and Applications, 27(1):99-106, 1979.

19. G. Leitmann. On the efficacy of nonlinear control in uncertain linear systems. Journal of Dynamic Systems, Measurement, and Control, 103(2):95-102, 1981.

20. P. McLane. Optimal stochastic control of linear systems with state-and control-dependent disturbances. IEEE Transactions on Automatic Control, 16(6):793-798, 1971. 
21. S. Olaru, J. A. De Doná, M. M. Seron, and F. Stoican. Positive invariant sets for fault tolerant multisensor control schemes. International Journal of Control, 83(12):2622-2640, 2010.

22. S. V. Raković, E. C. Kerrigan, K. I. Kouramas, and D. Q. Mayne. Invariant approximations of the minimal robust positively invariant set. IEEE Transactions on Automatic Control, 50(3):406-410, 2005.

23. S. V. Raković, E. C. Kerrigan, and D. Q. Mayne. Reachability computations for constrained discrete-time systems with state-and input-dependent disturbances. In Proceedings of the $42 \mathrm{nd}$ IEEE Conference on Decision and Control, volume 4, pages 3905-3910. IEEE, 2003.

24. V. Reppa, S. Olaru, and M. M. Polycarpou. Structural detectability analysis of a distributed sensor fault diagnosis scheme for a class of nonlinear systems. In Proceedings of the 9th IFAC SAFEPROCESS, Paris, France, 2015.

25. R. M. Schaich and M. Cannon. Robust positively invariant sets for state dependent and scaled disturbances. In Proceedings of the 54th IEEE Conference on Decision and Control, 2015.

26. M. M. Seron and J. A. De Doná. Robust fault estimation and compensation for LPV systems under actuator and sensor faults. Automatica, 52:294-301, 2015.

27. M. M. Seron, X. W. Zhuo, J. A. De Doná, and J. J. Martínez. Multisensor switching control strategy with fault tolerance guarantees. Automatica, 44(1):88-97, 2008.

28. F. Stoican and S. Olaru. Set-theoretic Fault-tolerant Control in Multisensor Systems. ISTE, John Wiley \& Sons, 2013.

29. J. L. Willems and J. C. Willems. Feedback stabilizability for stochastic systems with state and control dependent noise. Automatica, 12(3):277-283, 1976. 